\title{
La phénoménologie comme théorie transcendantale de l'histoire
}

\section{Ludwig Landgrebe}

Traducteur : Guillaume Fagniez et John Tryssesoone

\section{(Q) OpenEdition \\ Journals}

Édition électronique

URL : http://journals.openedition.org/alter/418

DOI : $10.4000 /$ alter.418

ISSN : 2558-7927

Éditeur :

Association ALTER, Archives Husserl (CNRS-UMR 8547)

\section{Édition imprimée}

Date de publication : 3 décembre 2017

Pagination : 251-273

ISBN : 978-2-9550449-3-3

ISSN : $1249-8947$

\section{Référence électronique}

Ludwig Landgrebe, « La phénoménologie comme théorie transcendantale de l'histoire », Alter [En ligne], 25 | 2017, mis en ligne le 01 décembre 2018, consulté le 10 décembre 2020. URL : http:// journals.openedition.org/alter/418; DOI : https://doi.org/10.4000/alter.418 


\section{LA PHÉNOMENOLOGIE COMME THÉORIE TRANSCENDANTALE DE L'HISTOIRE ${ }^{1}$}

Ludwig Landgrebe

Le titre de cet exposé énonce la thèse que nous allons tâcher de justifier: Si la phénoménologie doit être une philosophie transcendantale, elle est alors, pensée jusqu'à son dernier terme, une théorie transcendantale de l'histoire. Cette thèse laissera en suspens - entre parenthèses, pourrait-on dire - la question de savoir si la phénoménologie doit nécessairement être une théorie transcendantale. On le sait, cette question a été débattue parmi les élèves de Husserl dès la parution des Ideen et ce qu'il est convenu d'appeler le «tournant transcendantal » accompli par cet ouvrage. Vider cette querelle impliquerait cependant de clarifier au préalable la signification du terme « transcendantal » chez Husserl, et cela à partir de son œuvre ellemême. On ne peut se borner à lui attribuer la signification que la philosophie lui accordait avant Husserl. Ainsi, si notre propos se réfère bien évidemment à Husserl, il ne pourra être considéré comme une contribution à l'exégèse husserlienne que sous cette réserve. Les interprétations sont innombrables, entre lesquelles règne le plus complet désaccord. On a presque le sentiment que leur différence repose sur les passages et citations des textes de Husserl, eux-mêmes constitués de strates multiples, [18] qu'elles convoquent à leur appui. Cette querelle met en jeu quasiment tous les concepts opératoires fondamentaux de Husserl, dont aucun n'a pu démontrer son univocité. Néanmoins, la plupart des recherches récentes sur la phénoméno-

\footnotetext{
${ }^{1}$ Conférence inaugurale des Internationale phänomenologische Studientage [Journées internationales d'études phénoménologiques], Berlin, 23-27 mai 1974. Texte original paru dans les Phänomenologische Forschungen, vol. 3 («Phänomenologie und Praxis»), 1976, p. 17-47. Nous remercions les éditions Felix Meiner pour leur aimable autorisation de publier une traduction française de ce texte.
} 
logie husserlienne ont un point commun, celui de partir de l'œuvre tardive et des manuscrits de la dernière période, pour comprendre les travaux antérieurs comme autant d'étapes conduisant à ces dernières vues. Pour autant, elles ne se soumettent pas par là à une idée en vogue selon laquelle seule l'œuvre tardive d'un penseur énoncerait ses idées décisives et ultimes, à la lumière desquelles tous les travaux antérieurs ne devraient dès lors être envisagés que comme préparatoires. Bien plutôt, un tel principe méthodique a justement démontré ici sa fécondité en permettant de montrer que le développement de la pensée husserlienne ne connaît aucune rupture, mais correspond au contraire à une intention directrice présente dès le commencement, intention qui devait toutefois se déployer progressivement pour clarifier ce qu'elle visait initialement. Une telle situation correspond du reste entièrement à la conception husserlienne de l'intentionnalité et du rapport entre intention et remplissement comme passage de l'obscurité à la clarté. Ainsi, la démarche consistant à partir du « dernier » Husserl pour argumenter contre le premier, démarche qui a été celle de toutes les recherches récentes un tant soit peu fécondes, a prouvé sa légitimité par les succès dont elle a été couronnée. C'est pourquoi toute confrontation avec le projet husserlien de philosophie transcendantale qui, de manière précipitée, entreprendrait d'évaluer celui-ci à partir d'objectifs liés à d'autres perspectives philosophiques, est nécessairement vouée à l'échec. Il faut au contraire commencer par penser sa propre position, avec toutes les possibilités dont elle est grosse, jusqu'à son terme ; après quoi seulement il devient possible de discerner [19] où se situent éventuellement les limites d'une réflexion philosophico-transcendantale et où il est nécessaire de dépasser celles-ci en empruntant une autre démarche.

S'il devait s'avérer que les réflexions tardives de Husserl contiennent elles-mêmes l'indication de ces limites, sa philosophie pourrait alors légitimement prétendre au titre de «philosophie transcendantale » : "transcendantal » au sens d'un mode et d'une voie de connaissance qui rendent possible de fixer soi-même ses propres limites de manière critique. Ce concept de «transcendantal » correspondrait également aux explications données dans un manuscrit manifestement rédigé après la Krisis, et qui jette un regard rétrospectif sur cette œuvre, notamment sur le paragraphe sur Galilée. Husserl y évoque certaines difficultés sensibles de l'œuvre, en l'occurrence le fait $\mathrm{qu}^{\prime}$ " au sein d'un tel développement historique, un nouveau style de questionnement philosophique et une nouvelle méthode philosophique se font progressivement jour ». Son sens général implique «l'entrelacement de la recherche historique et de la recherche systématique 
motivée par cette dernière, entrelacement d'emblée mis en œuvre dans la réflexivité particulière dans laquelle seule peut se mouvoir l'auto-réflexion du philosophe, en tant que celui-ci se trouve dans une situation où il ne peut présupposer aucune philosophie donnée, $q u^{\prime}$ elle soit ou non la sienne $»^{2}$.

Formellement, l'acception husserlienne du terme "transcendantal » partage donc avec son acception kantienne le sens de «critique »: critique du recours à des concepts reçus, question du quid juris de leur usage en tant que moyens d'expliciter notre expérience. En ce sens, «transcendantal » ne signifie rien d'autre que l'opposition absolue à toute forme de [20] dogmatisme: non seulement opposition au dogmatisme de la métaphysique que Kant a pris pour cible, mais également critique des implications métaphysiques cachées dans les positions antimétaphysiques modernes; en termes généraux, donc, critique de l'usage incontrôlé de tous les concepts philosophiques reçus au moyen desquels on entend produire une compréhension de notre monde et in fine une explication globale du monde. En ce sens, la philosophie transcendantale est tout à la fois une critique de l'usage linguistique et - dans la mesure où elle se déploie comme une confrontation avec des théories métaphysiques et antimétaphysiques elle-même une théorie. Cependant, dans la mesure où les tentatives d'explication du monde ont toujours été, en leur sens originel, davantage que de "simples » théories, dans la mesure où elles ont aussi tenté de comprendre le monde pour y trouver un juste chemin pour la vie, elles ont toujours eu pour corollaires des principes pratiques de l'action. Pour cette raison, la critique transcendantale de ces théories est toujours en même temps critique des principes d'action qui en découlent. Une telle articulation se donne à voir dès l'élaboration originelle du concept de theôria chez Aristote, et Husserl lui-même a finalement compris sa phénoménologie transcendantale comme une « critique de la vie ».

Cela devrait suffire pour une compréhension provisoire du concept de «transcendantal» et du sens de la phénoménologie comme théorie transcendantale. Par là, nous n'avons encore rien dit sur l'histoire comme thème de cette théorie transcendantale. On sait que le terme « histoire» a au moins deux significations: on peut y entendre aussi bien la res gestae que l'historia rerum gestarum, la science de l'histoire. Or, avec la thèse avancée plus haut, nous

${ }^{2}$ Cf. E. Husserl, Die Krisis der europäischen Wissenschaften und die transzendentale Phänomenologie, Hua VI, W. Biemel (éd.), La Haye, Martinus Nijhoff, 1976, p. 363-364 [La crise des sciences européennes et la phénoménologie transcendantale, trad. G. Granel, Paris, Gallimard, 1976, p. 401-402]. 
n'entendons pas soutenir que la phénoménologie transcendantale [21] doive être examinée ici dans la mesure seulement où elle est une théorie de la connaissance historique, ou même qu'elle se réduise en général à une telle théorie, c'est-à-dire une théorie de ce qu'on a pu appeler les "sciences de l'esprit ». En effet, lorsqu'on envisage une telle théorie comme une tâche philosophique, on désigne par là un degré de réflexion subordonné à la réflexion philosophico-transcendantale, devant d'abord être fondé par elle. La phénoménologie transcendantale a affaire au premier chef à la question « qu'est-ce que l'histoire? », c'est-à-dire à la question des conditions transcendantales de la possibilité de quelque chose comme l'histoire, laquelle peut ensuite devenir objet d'investigation scientifique. C'est en ce sens que Husserl a interrogé $\mathrm{l}^{\prime}$ «a priori de l'histoire ». Mais, comme nous le verrons, cet a priori revêt une forme tout à fait singulière.

Une considération relevant de la philosophie transcendantale ne peut donc pas elle-même admettre purement et simplement comme quelque chose de donné les divisions de domaines entre les diverses sciences particulières. Ces divisions sont en effet nées principalement des nécessités pratiques propres à ces connaissances et sont aujour$\mathrm{d}$ 'hui largement mises en cause. C'est pourquoi nous ne pouvons pas non plus nous fonder sur le projet d'ontologies régionales exposé par Husserl dans les Ideen II qui servit encore de point de référence à Heidegger dans Sein und Zeit. Ce projet se contentait en effet luimême d'adopter comme quelque chose de donné les divisions de domaines alors admises entre les sciences. Il est issu d'une considération statique. Or c'est seulement en tant que phénoménologie génétique que la phénoménologie transcendantale a atteint son concept pleinement développé - ce qui n'a pas besoin d'autre justification ici ${ }^{3}$. La phénoménologie en tant que théorie transcendantale de l'histoire [22] ne peut donc être que la phénoménologie génétique. Nous verrons que, si les résultats de cette dernière ont évidemment aussi une signification pour la théorie de la connaissance scientifique, le terme $\mathrm{d}^{\prime}$ « histoire » recouvre cependant davantage que la notion de res gestae. Le sens en lequel il est question d'histoire dans notre thèse doit donc d'abord être laissé en suspens.

Une théorie transcendantale de l'histoire présuppose également une théorie satisfaisante de l'intersubjectivité. Celle-ci ne peut ellemême être pleinement développée que sous la forme d'une théorie

${ }^{3}$ Cf. A. Aguirre, Genetische Phänomenologie und Reduktion. Zur Letztbegründung der Wissenschaft aus der radikalen Skepsis im Denken E. Husserls, Phaenomenologica 38, La Haye, Martinus Nijhoff, 1970, p. $153 \mathrm{sq}$. 
génétique. Il a toutefois été montré que la théorie husserlienne de l'intersubjectivité exposée dans la cinquième Méditation Cartésienne a échoué précisément du fait que Husserl tente de la développer sous une forme statique ${ }^{4}$. Même les tentatives plus tardives de Husserl, aujourd'hui publiées dans le volume XV des Husserliana, ne lui ont pas permis de parvenir à bon port. Certaines de ces contributions contiennent toutefois l'indication de la manière dont une telle lacune peut être comblée. Il nous faudra revenir sur ce point.

Après ces remarques introductives, nous abordons la justification de notre thèse qui, de manière un peu plus complète, peut s'énoncer dorénavant ainsi : la phénoménologie transcendantale génétique est en tant que telle une théorie transcendantale de l'histoire. Nous nous rattacherons ici à deux pensées de Husserl, qui au premier abord pourraient sembler incompatibles entre elles. La première concerne la caractérisation de $l^{\prime}$ «ego»dont les effectuations constitutives du monde forment le thème de la phénoménologie. La réduction phénoménologique reconduit à la certitude apodictique que ce Je a de lui-même. Dans cette réflexion, le Je est immédiatement présent à lui-même. Il est, dans cette présence à soi, apodictiquement certain de ses fonctions constitutives [23] et peut, dans une variation eidétique, reconnaître ces fonctions comme appartenant nécessairement à tout Je pensable en général. Depuis le début, Husserl a certes toujours souligné qu'une telle réflexion était accomplie par un Je existant facticement ; mais, conformément à la théorie de l'essence développée dès les Ideen, un fait singulier est contingent au regard de son essence. À partir de 1931, cependant, dans des manuscrits pour partie encore inédits, cette thèse se voit inversée: "L'eidos Je transcendantal est impensable sans un Je transcendantal comme factice $»^{5}$. En effet :

«moi » qui modifie en pensée, moi qui me libère de l'effectivité factice, je suis apodictiquement le Je de l'effectivité factice, et je suis, en particulier en tant que Je pensant et voyant eidétiquement, le Je de capacités que j'ai acquises facticement. Les possibilités imaginaires en tant que variantes de l'eidos ne flottent pas librement en l'air, mais elles sont constitutivement rapportées à moi-même dans mon factum, avec mon présent vivant que je « vis » facticement ${ }^{6}$.

\footnotetext{
${ }^{4}$ K. Held, « Das Problem der Intersubjektivität und die Idee einer phänomenologischen Transzendentalphilosophie », in Perspektiven transzendental-phänomenologischer Forschung (für Ludwig Landgrebe zum 70. Geburtstag), U. Claesges et K. Held (éd.), Phaenomenologica 49, La Haye, Martinus Nijhoff, 1927, p. 3-60.

${ }^{5}$ E. Husserl, Zur Phänomenologie der Intersubjektivität. Texte aus dem Nachlass. Dritter Teil : 19291935, Hua XV, I. Kern (éd.), La Haye, Martinus Nijhoff, 1973, Nr. 22, p. 385.

${ }^{6}$ Ms. K III 12, p. 34 sq. [Voir à présent E. Husserl, Die Krisis der europäischen Wissenschaften und die transzendentale Phänomenologie. Ergänzungsband. Texte aus dem Nachlass 1934-1937, Hua XXIX, R. N.
} 
Par conséquent : "La structure apodictique de l'effectivité transcendantale n'est pas, en vertu de ce pouvoir de modifier en pensée, une structure qui serait contingente - un fait contingent qui aurait un cadre d'essence contenant d'autres possibilités qui "auraient tout aussi bien pu exister" $\gg^{7}$. De sorte que ce Je que je suis moi-même doive être désigné comme un factum absolu:

Je suis le factum originaire dans cette démarche de questionnement à rebours [...] l'absolu qui a en lui-même son fondement et, dans son être sans fondement, sa nécessité absolue en tant que la "substance absolue » une. Sa nécessité n'est pas une nécessité d'essence qui laisserait ouvert quelque chose de contingent. Toutes les nécessités d'essence sont bien plutôt des moments [24] de son factum [...] ses manières de se comprendre ou de pouvoir se comprendre lui-même ${ }^{8}$.

«L'absolu que nous dévoilons est un "fait" absolu»9.

Ces thèses rendent au premier abord un son étrange. Ne transgressent-elles pas les limites d'une philosophie transcendantale en passant à une métaphysique de l'absolu qui ne serait pas sans rappeler la «philosophie positive» de Schelling ? Une telle interprétation fut déjà récusée en son temps par Eugen Fink ${ }^{10}$. Celui-ci eut connaissance de ces pensées dès l'époque de leur apparition (en 1931), époque à laquelle Husserl travaillait au projet, finalement abandonné, de révision du texte allemand des Méditations cartésiennes. Selon Fink, il ne s'agirait pas ici d'un passage à une métaphysique spéculative, mais bien plutôt de "concepts dus à l'embarras de situations philosophiques limites » qui se présentent dans l'effort inlassable de "prendre sur le fait », pour ainsi dire, dans une analyse réflexive, 1 '«effectuation de la vie de la conscience transcendantale ${ }^{11}$. Si tel est le cas, cela vient alors confirmer la légitimité de la prétention de la phénoménologie au titre de «transcendantal ». Par là, la phénoménologie aurait démontré qu'elle est en mesure de se rendre à elle-même évidentes ses propres limites de manière critique.

Pour parvenir ici à la clarté, nous allons confronter cette thèse sur l'absoluité de l'ego avec une seconde thèse, relative à l'histoire, qui se

Smid (éd.), Dordrecht/Boston/Londres, Kluwer, 1993, Nr. 8, p. 85 ; «Variation et ontologie », trad. C. Lobo, Annales de phénoménologie, n5, 2006, p. 225.]

${ }^{7}$ Idem.

${ }^{8}$ Hua XV, Nr. 22, p. 386.

${ }^{9}$ Hua XV, Beilage XXIII, p. 403.

${ }_{10}$ Cf. E. Fink, «Die Spätphilosophie E. Husserls in der Freiburger Zeit », in Edmund Husserl 18581959. Recueil commémoratif, Phaenomenologica, ${ }^{\circ} 4$, La Haye, Martinus Nijhoff, 1959, p. 99-115 [ «La philosophie tardive de Husserl dans la période de Fribourg », in E. Fink, Proximité et distance. Essais et conférences phénoménologiques, trad. Kessler, Grenoble, Jérôme Millon, 1994, p. 169-187].

11 Ibid., p. 114 [trad. p. 185]. 
trouve formulée dans le paragraphe final d'un manuscrit de recherche intitulé : "Réduction phénoménologique et justification absolue ». Cette thèse s'énonce ainsi : «L'histoire est le fait majeur de $l^{\prime}$ être absolu $»^{12}$. Dans le manuscrit de Husserl, ce paragraphe final a reçu [25] pour sous-titre: "Monadologie». À cet égard, nous pouvons commencer par formuler deux remarques :

$1^{\circ}$ ) Le manuscrit date du début des années vingt. Il est donc faux de considérer que Husserl n'aurait découvert l'importance de l'histoire pour la phénoménologie que dans la dernière phase de sa pensée. Cela nous contraint bien plutôt à comprendre cette découverte, non comme une rupture dans sa pensée, mais au contraire comme une conséquence de sa première percée dans la philosophie transcendantale.

$\left.2^{\circ}\right)$ Ce manuscrit donne une indication sur le contexte dont est issue la thèse husserlienne sur l'histoire, à savoir celui de la monadologie. Les interprètes ont accordé jusqu'ici trop peu d'attention à cet intitulé, et ont pour cette raison manqué le problème que recelait cette réception d'un terme leibnizien. Il s'agit en effet d'une réception dans laquelle le sens de ce terme se voit transformé. Si les monades leibniziennes n'ont pas de fenêtres, c'est en revanche le cas des «monades» dont parle la phénoménologie transcendantale pour désigner les ego transcendantaux. C'est en ce sens que Husserl parle également de l'intersubjectivité transcendantale comme du « tout des monades ».

À cela se rattachent deux questions :

$1^{\circ}$ ) De quel droit applique-t-on à $l^{\prime}$ « ego transcendantal », qui dans sa présence apodictiquement certaine pour lui-même est un «fait absolu », le titre de «monade »?

$2^{\circ}$ ) De quelle manière les deux thèses sur $1^{\prime}$ «absolu » sont-elles [26] compatibles entre elles, c'est-à-dire de quelle manière s'entre-appartiennent-elles en se complétant mutuellement, et qu'est-ce qui en résulte pour la compréhension de la conception husserlienne de l'absolu et pour le concept phénoménologico-transcendantal d'histoire?

Manifestement, ces deux questions ne peuvent être traitées séparément. Leur entre-appartenance constitue précisément le problème

\footnotetext{
12 E. Husserl, Erste Philosophie (1923/24). Zweiter Teil : Theorie der phänomenologischen Reduktion, Hua VIII, R. Boehm (éd.), La Haye, Martinus Nijhoff, 1959, Beilage XXXII, p. 506. Pour une interprétation détaillée de cette thèse, voir L. Landgrebe, "Meditation über Husserls Wort „Die Geschichte ist das große Faktum des absoluten Seins"”, Tijdschrift voor Filosofie, n 36, 1974, p. 107-126 ["Méditation sur le mot de Husserl: "L'histoire est le fait majeur de l'être absolu" », trad. G. Fagniez, Philosophie, n 110, 2011, p. 31-45]. Les développements qui suivent peuvent être considérés comme une variation, en partie complémentaire et en partie améliorée, sur ce thème.
} 
phénoménologico-transcendantal de l'histoire qu'il s'agit à présent de déployer, du moins dans ses grandes lignes. À titre de préambule, nous indiquerons d'abord le sens que revêt l'introduction du concept de monade pour la théorie de la science historique.

À cet égard, il convient de rappeler que l'idée centrale de la philosophie de l'histoire de Herder fut manifestement elle-même inspirée par la monadologie leibnizienne. Cette idée est celle de l'absolue individualité et unicité de tout ce qui est historique. Ce qui est historique possède sa signification absolue en lui-même et non par les seuls effets qui en découlent. Avec cette idée, Herder entendait critiquer l'idée de progrès des Lumières, en particulier celle de Voltaire. Il prépara ainsi le terrain pour la science moderne de l'histoire, guidée par l'intérêt pour l'être individuel. Dans ses conférences prononcées devant le roi Maximilien de Bavière sur les époques de l'histoire moderne, Leopold von Ranke reprit presque à la lettre la thèse de Herder. Il en fit le credo de la science historique, à l'aune duquel seul le sens de l'objectivité exigée par cette science devrait être entendu. Cette exigence de Ranke fut combattue par l' « histoire politique », en particulier par Gervinus. Selon celui-ci, la science historique aurait pour tâche de servir les intérêts du jour, et au premier chef les intérêts définis par l'auto-affirmation de la nation. Ranke s'opposa fermement à cette thèse, [27] dont il voyait les conséquences relativistes et avec elles la fin de l'histoire en tant que science. Ce débat montre que ce n'est pas l'idée d'individualité, mais au contraire sa violation, qui conduit au relativisme et qui dès lors compromet l'accès à une vérité de l'histoire. Cette indication devrait suffire à montrer que cette querelle entre Ranke et Gervinus n'a pas simplement un intérêt historique, mais qu'au contraire un problème fondamental de la connaissance historique y est en jeu, problème qui est encore débattu aujourd'hui. Au début des années cinquante, la Historische Zeitschrift a fait état d'une discussion entre historiens américains autour des principes méthodiques selon lesquels il convenait de rendre compte de l'attaque japonaise sur Pearl Harbour. Cette discussion mettait aux prises exactement les mêmes positions que celles incarnées en leur temps par Ranke et Gervinus. On peut aussi rappeler le traitement notoirement réservé à l'histoire par les bolchéviques, pour lesquels l'histoire doit sans cesse être réécrite selon les intérêts du parti. Le problème est ainsi celui de savoir si la vérité de ce que l'histoire transmet se réduit à ce qui a un sens au regard du présent et de ses intérêts, ou s'il faut au contraire, avant toute autre considération, l'apprécier dans son individualité, "tel que cela s'est réellement passé ». Consciente de ce problème, l'école néokantienne du Sud-Ouest a cherché, dans sa 
théorie de l'histoire, à distinguer la connaissance « idiographique » de cette dernière de la connaissance "nomothétique " propre aux sciences de la nature. Il nous faut renoncer ici à entrer dans une discussion critique de la solution qu'a tenté d'apporter la théorie néokantienne au problème de l'individualité grâce au concept de "référence à la valeur ", [28] même si une telle discussion conserverait toute son actualité. Cette théorie de la référence à la valeur fait aujourd'hui encore sentir ses effets dans les débats contemporains relatifs à la neutralité axiologique dans le champ de la théorie sociale.

Avec cette indication, il ne s'agit pas pour nous de prétendre que la «découverte» de l'histoire par Husserl aurait été influencée de quelque manière que ce soit par le développement de la théorie de l'histoire depuis Herder. Rien n'indique qu'il y ait seulement prêté attention, ni a fortiori qu'il se soit particulièrement penché sur Herder. Il a été conduit à la signification du "fait originaire » de l'individualité pour l'histoire par le développement conséquent de sa propre voie, et il est possible de montrer qu'on parvient, en suivant cette voie, à donner à la vérité historique un fondement plus solide que celui procuré par la théorie néokantienne de l'histoire. Cette indication entendait simplement mettre en évidence que l'adoption du terme leibnizien de «monade » pour désigner le fait absolu de l'ego transcendantal en sa présence vivante à soi n'est pas une simple "expression due à l'embarras »: avec lui est pointé le problème central de la fondation de la vérité, tel qu'il se pose encore aujourd'hui aux historiens. Or, ce problème ne concerne pas seulement la théorie de la science historique, mais il porte également sur le rapport que les hommes, qui appartiennent toujours à une certaine époque et à un certain groupe, entretiennent déjà avec leur histoire avant toute connaissance scientifique de cette dernière. Un tel problème revêt une actualité particulière à une époque menacée par la perte d'histoire et les tendances à s'en détourner.

Passons à présent à la seconde question, qui porte sur l'articulation entre les deux thèses de l'absoluité de l'« ego » et de l'absoluité de [29] l'histoire, ainsi que sur le concept phénoménologico-transcendantal d'histoire qui procède de cette articulation. Nous avons rappelé qu'aussi bien la philosophie de l'histoire de Herder que la "monadologie » de Husserl se réfèrent au concept leibnizien de monade. L'arrière-plan historique de la monadologie leibnizienne est l'idée, caractéristique de la Réforme, d'un rapport immédiat de tout individu avec Dieu. Cette immédiateté implique que chaque monade humaine ne dépend que de Dieu, son Créateur. Elle doit donc être en elle-même close, "sans fenêtres", si bien que seule une harmonie préétablie peut garantir la possibilité d'un commercium des monades 
entre elles. Une telle fondation métaphysique de l'idée de monade et de son commercium est toutefois refusée à la phénoménologie transcendantale. C'est moyennant son mode particulier de réflexion sur la subjectivité que la phénoménologie doit parvenir à justifier l'introduction du concept de monade, de telle sorte que la thèse de son absoluité conserve ses droits mais que, en même temps, puisse subsister son commercium avec d'autres. Pour cela, il faudrait que les monades soient conçues comme n'étant pas dépourvues de fenêtres. Il incombe donc aux analyses phénoménologiques de situer le point où peuvent être trouvées ces "fenêtres ». Aussi est-il besoin d'une théorie de l'intersubjectivité, comme nous l'avons déjà dit, qui soit capable de répondre à cette question.

Afin de saisir plus nettement le problème, nous pouvons commencer par faire droit à une objection que la référence à la provenance historique de l'idée de monade paraît justement rendre inévitable : le style d'une telle réflexion n'est-il pas lui-même un résultat de l'histoire moderne de la pensée, avec ses présupposés spécifiques remontant à Descartes, [30] et dont les racines plongent profondément dans l'histoire de la pensée occidentale? On sait que ce style de réflexion n'a pas été développé par d'autres cultures. Le concept de singularité et d'unicité et, en ce sens, d'absoluité de chaque être humain, c'est-à-dire de chaque être pourvu de faculté de pensée et de réflexion, est étranger à ces cultures. Dès lors, un tel concept n'est-il pas le produit d'un développement culturel et d'une histoire entièrement déterminés, spécifiquement occidentaux? Ne s'agit-il pas d'une idée qui ne pouvait être formulée que dans des conditions historiques entièrement déterminées? Or, un simple fait historiquement conditionné peut-il conduire à la vision apodictique de quelque chose $\mathrm{d}$ 'inconditionnellement universel et nécessaire ? Cette question fut déjà posée par Lessing, qui y répondit par la négative: "Les faits historiques ne peuvent jamais être pris pour preuves de vérités éternelles ${ }^{13}$. Ainsi, le recours réflexif au «Je suis », en tant qu'il est historiquement conditionné, peut-il conduire à une vision évidente de ce que nous sommes tous, en tant que membres de $l^{\prime}$ « humanité »? Telle est au fond la question que Husserl s'est posée dans la Krisis : la question de la portée universelle de ce style de réflexion à l'origine spécifiquement européen.

Cette question peut être formulée de la façon suivante. La voie de la réflexion phénoménologique conduit Husserl à l'idée de l'absoluité

\footnotetext{
${ }^{13}$ Citation approximative d'une sentence célèbre de Lessing tirée de Über den Beweis des Geistes und der Kraft, 1777. [N. d. T.]
} 
de $l^{\prime}$ « ego transcendantal» en tant que monade, et pour ce qui concerne le rapport de cet ego à l'histoire, à une remarque qui doit être considérée comme l'ultime réponse de Husserl à la question de $l^{\prime}$ « $a$ priori » de l'histoire : le monde est historique seulement "par l'historicité intrinsèque de chaque sujet singulier, et de chacun en tant que personne singulière dans son historicité intrinsèque en communauté avec $d^{\prime}$ autres personnes ${ }^{14}$. [31] «Historicité intrinsèque »a chez Husserl le sens d'une caractérisation de la qualité monadique d'unicité de chaque sujet personnel singulier. Elle est ainsi $l^{\prime}$ «a priori», c'est-à-dire la structure sur la base de laquelle seulement il y a pour nous « $l$ 'histoire ». Dans quelle mesure cependant un tel rapport est-il valable dans une universalité apodictique pour tout sujet personnel, et dans quelle mesure permet-elle finalement de parler d'une « histoire de l'humanité »? Et pourquoi cette proposition ne peut-elle être inversée, s'il est vrai par ailleurs que «l'histoire est le fait majeur de l'être absolu »? Inversée, la proposition énoncerait : l'histoire est le présupposé a priori de l'historicité intrinsèque de chaque sujet égoïque singulier. Cela ne serait cependant possible que si la "monade » se trouvait déjà préalablement inscrite dans l'enchaînement de l'histoire. Exprimé dans le jargon moderne, cela signifierait que l'historicité intrinsèque n'est rien d'autre que l'internalisation de l'histoire. Ce n'est que sous certaines conditions historiques entièrement déterminées qu'une telle internalisation serait possible et qu'un concept $\mathrm{d}^{\prime}$ « historicité » pourrait se constituer. Mais dès lors, aucune valeur contraignante ne reviendrait à un tel concept au-delà du domaine circonscrit par la culture occidentale.

Ce n'est que si l'on peut montrer comment la réflexion phénoménologico-transcendantale est en mesure de trancher cette alternative de façon contraignante que l'on pourra comprendre l'entre-appartenance des deux thèses de Husserl, et que l'affirmation selon laquelle la phénoménologie transcendantale génétique est une théorie transcendantale de l'histoire pourra à son tour être fondée. [32] Pour ce faire, il convient à présent de retracer brièvement la voie réflexive qui a conduit Husserl à ces deux thèses. Il s'agit d'examiner plus précisément la forme spécifique de réflexivité qui se caractérise, selon la formule citée plus haut, par l'entrelacement du questionnement historique et du questionnement systématique motivé par ce dernier.

La phénoménologie transcendantale, sous sa forme pleinement développée de phénoménologie génétique, est également désignée

\footnotetext{
${ }^{14}$ Hua VI, p. 381, note 1 [La crise des sciences européennes et la phénoménologie transcendantale, op. cit., p. 421, note 1].
} 
par Husserl comme une recherche d'origine, et même finalement comme une "archéologie » de la conscience, dans la mesure où elle thématise génétiquement la «couche enfouie » de la pré-constitution passive. L'origine de toutes les représentations de l'expérience et de la connaissance réside dans les fonctions constitutives appartenant à la subjectivité, lesquelles sont accessibles à la réflexion. Pour Kant déjà, la philosophie transcendantale était guidée par la question de l'origine. Celui-ci distinguait origine rationnelle et origine temporelle $^{15}$. Mais cette distinction est rejetée par Husserl: "Le dogme dominant de la séparation de principe entre élucidation gnoséologique et explication historique [...], entre origine gnoséologique et origine historique, est $[. .$.$] fondamentalement erroné { }^{16}$. Mais quelle forme particulière de réflexivité permet de justifier cette assertion? Cette forme n'est pas recensée dans la table des concepts usuels de réflexion établie par Hans Wagner dans son article «Réflexion » du Handbuch philosophischer Grundbegriffe ${ }^{17}$. La réflexion au sens husserlien y est certes mentionnée au titre de la "réflexion constitutive ». Cette dernière a le sens d'une reconduction réflexive du noème, en tant que produit, aux actes qui le produisent et le constituent. Une telle caractérisation correspond parfaitement à [33] la compréhension qu'a Husserl de sa propre démarche. Celle-ci est toujours orientée sur le schéma de la constitution d'objet, une constitution dans laquelle l'objet se forme comme unité synthétique de ses modes d'apparition, et c'est sur ce modèle que Husserl tente de comprendre l'autoconstitution du Je en tant que pôle identique dans la multiplicité de ses actes constituants. Ainsi comprise, la réflexion est toujours une " prise de conscience après coup », un se-retourner ultérieur sur les fonctions déjà mises en œuvre ${ }^{18}$. Il s'agit là toutefois $\mathrm{d}^{\prime}$ un concept statique de réflexivité. Husserl a de facto dépassé de bonne heure les limites de la démarche statique à travers ses analyses génétiques. Mais il n'est pas revenu sur ce dépassement lui-même et ne l'a pas thématisé dans sa signification systématique. Nous allons tenter d'en donner une esquisse simplifiée.

\footnotetext{
${ }^{15}$ I. Kant, Religion innerhalb der Grenzen der bloßen Vernunft, Ak VI, p. 39 [La religion dans les limites de la simple raison, trad. A. Philonenko, in CEuvres philosophiques III, F. Alquié (éd.), Paris, Gallimard «La Pléiade», 1986, p. 54].

${ }^{16}$ Hua VI, p. 379 [La crise des sciences européennes et la phénoménologie transcendantale, op. cit., p. 418-419].

${ }_{17}$ Cf. H. Krings, H. M. Baumgartner, C. Wild (éd.), Handbuch philosophischer Grundbegriffe, 6 vol., Munich, Kösel, 1973-1974.

${ }_{18}$ Cf. L. Landgrebe, Der Weg der Phänomenologie. Das Problem einer ursprünglichen Erfahrung, Gütersloh, Mohn, 1963, p. 192 sq.
} 
La réflexion statique ne peut reconduire à l'origine de notre familiarité avec nous-mêmes et avec nos fonctions constitutives, familiarité qui précède toute réflexion sur des actes déjà accomplis. Pour comprendre ce mode particulier de réflexivité en tant que familiarité préalable avec nousmêmes, il suffit de considérer la manière dont, dans la vie quotidienne, nous sommes motivés à interrompre nos accomplissements d'actes pour réfléchir sur leur succès ou leur insuccès. Cela arrive le plus souvent lorsque nous nous heurtons soudain à des difficultés dans l'accomplissement d'une action. L'accomplissement naïf est alors interrompu par une prise de conscience qui, exprimée verbalement, se formulerait ainsi : "Pourquoi n'en as-tu pas été capable?» Aussi la réflexion est-elle toujours en premier lieu un retour sur ce que nous pouvons. Mais ce que nous pouvons, seul l'exercice de notre pouvoir est à même de nous l'apprendre. C'est ce que Husserl luimême pressent parfaitement [34] lorsque, dans son analyse du pouvoir kinesthésique dans Ideen II, il dit que « le "je meus" [...] précède le "je peux" ${ }^{19}$. Cette description vaut déjà pour le niveau du développement pré-langagier de l'enfant, où le mouvement intentionnel des membres du corps procède de l'imitation et de la répétition. Dans cet exercice, à travers son succès et son insuccès, l'enfant s'éprouve très tôt comme centre et source du mouvement spontané par lequel il peut agir (prendre, repousser, etc.) dans son monde environnant. Cette expérience est l'origine de toutes les représentations de forces efficientes. C'est en elle que nous faisons originairement l'expérience d'un « parce que - donc ». D'un point de vue génétique, le fait que ce centre du mouvement spontané et des actions se pose comme un Je face à un Tu est second. Auparavant il est déjà cette existence individuelle et se «sait » comme tel par l'expérience qu'il a du pouvoir de disposer de son corps, grâce la capacité qu'il a progressivement acquise de maîtriser sa motricité. C'est cette expérience qui lui ouvre le premier accès à son monde environnant.

On pourrait naturellement se demander si cette sorte de familiarité pré-langagière avec soi-même comme centre du pouvoir de se mouvoir spontanément est spécifiquement humaine, ou si elle ne doit pas plutôt être également attribuée aux animaux. Ne vaut-elle pas pour tout organisme en tant qu'unité capable de se diriger soi-même ? À cela, on peut seulement répondre que, s'il va de soi que les animaux

\footnotetext{
${ }^{19}$ E. Husserl, Ideen zu einer reinen Phänomenologie und phänomenologischen Philosophie. Zweites Buch: Phänomenologische Untersuchungen zur Konstitution, Hua IV, M. Biemel (éd.), La Haye, Martinus Nijhoff, 1952, p. 261 [Idées directrices pour une phénoménologie et une philosophie phénoménologique pures. Livre second: Recherches phénoménologiques pour la constitution, trad. É. Escoubas, Paris, PUF, 1982, p. 354].
} 
fonctionnent comme centres de mouvement, ils ne se savent cependant pas tels. C'est en ce sens que Husserl parle, en référence à Leibniz, de "monades endormies». Engager une discussion de ce problème nous conduirait toutefois trop loin.

Ce que nous entendions simplement dire, en indiquant cette origine de tout savoir portant sur un pouvoir, c'est que, sans cette familiarité originaire avec [35] le pouvoir kinesthésique, il ne pourrait $\mathrm{y}$ avoir aucun niveau supérieur de réflexivité, cette dernière ayant toujours le sens d'une réflexion sur le pouvoir, sur son espace de jeu et ses limites. Ce pouvoir de se mouvoir est la forme la plus élémentaire de la spontanéité; si bien qu'une théorie de l'action qui ne le prendrait pas pour point de départ ne pourrait aboutir qu'à un concept global et abstrait de l'action. Même l'acte de parler, dans lequel la faculté de penser se voit pour la première fois articulée, a la structure du mouvement kinesthésique. Par conséquent, ces fonctions kinesthésiques (sans lesquelles il $n^{\prime} y$ aurait pas même de constitution du temps) forment la dimension la plus profonde de la subjectivité transcendantale, la véritable sphère originaire; de sorte que le corps aussi, en tant qu'il fonctionne, n'est pas un simple objet constitué, mais lui-même constituant, en tant que condition transcendantale de la possibilité de tout niveau supérieur de conscience et de son caractère réflexif ${ }^{20}$.

Husserl n'a pas tiré explicitement cette conséquence. Mais lorsqu'il évoque la «face nature » de la subjectivité, et le fait que la subjectivité transcendantale ne peut être désignée comme un Je que per aequivocationem, cela indique qu'il ne voulait pas comprendre la "nature » comme l'autre de la subjectivité, mais au contraire comme l'ultime soubassement de cette subjectivité même. La nature ainsi entendue n'est pas la nature objectivée comme ensemble de l'existence réale " en dehors de nous», mais la nature telle que nous en faisons immédiatement l'expérience dans la familiarité avec nos fonctions corporelles. Néanmoins, si cette maîtrise de nos fonctions kinesthésiques est bien ce qui nous ouvre le premier accès à notre monde, il serait faux de voir dans notre corps le principe d'individuation. Il ne peut être un tel principe que pour autant que quelqu'un sache ce corps comme étant son corps, dont il peut disposer dans certaines limites. C'est ce qu'a également vu Husserl, qui avance que «la découverte du Mien précède la découverte du Je $»^{21}$. Mais il n'en a

\footnotetext{
${ }^{20}$ Cf. L. Landgrebe, "Reflexionen zu Husserls Konstitutionslehre », Tijdschrift voor Filosofie, $\mathrm{n}^{\circ} 36,1974$, p. $466-482$.

${ }^{21}$ Hua IV. [Le passage dont cette citation est extraite n'a pu être retrouvé. [N. $\left.\left.d . T.\right]\right]$
} 
pas tiré les conséquences qui en résultaient pour la compréhension du sens de l'absoluité de l'« ego transcendantal » et pour la vision du sens avant tout pratique de la réflexion en tant que réflexion sur le pouvoir.

Le pouvoir de se mouvoir est la fonction transcendantale la plus profonde avec laquelle se constitue également le temps. Ce n'est donc pas sans raison si la première analyse détaillée du temps, celle d'Aristote, a été menée dans le cadre d'un questionnement sur le mouvement ${ }^{22}$. C'est dans l'expérience que nous faisons de l'exercice de ce pouvoir que s'ouvre à chaque fois, pour moi comme pour l'autre, le Là imprescriptible comme certitude apodictique de notre présent fonctionnel vivant. C'est uniquement de ce Là que le concept de factum absolu reçoit son sens :

D'un côté, le Je fonctionnant ultimement est, avant tout exercice de la libre variation et de l'idéation d'une "égoïté en général », et avant la position d'un nunc stans omni-temporel, déjà là ; le Je se heurte pour ainsi dire à ce «Là » qu'il est lui-même. D'un autre côté, il faut dire que ce Là n'est pas « pré-donné » de la même manière que des stimuli sensibles précèdent constitutivement les expériences de niveau supérieur $^{23}$.

En d'autres termes, pour recevoir des stimuli sensibles, il est déjà besoin de ce Là. Ce qui doit être compris correctement : lorsque nous parlons de notre Là, cela implique que nous visons aussi par ce démonstratif un Là à sa place temporelle propre, précédé et suivi par d'autres Là. Mais cette manière de parler procède d'une réflexion après coup dans laquelle ce Là est déjà objectivé comme un Là parmi d'autres, auxquels correspond, persistant en chacun d'eux, le [37] « Je stable et durable ». C'est également cette réflexion que la dialectique hégélienne de la certitude sensible met en branle, mais en vue de montrer que cette prétendue immédiateté est elle-même médiatisée. Ici, il s'agit au contraire de montrer que ce présent fonctionnel vivant est une certitude apodictique imbiffable, un factum au-delà duquel on ne peut remonter, et est donc, en ce sens, immédiat et absolu, en tant que factum absolu, qui est la condition transcendantale la plus

\footnotetext{
${ }^{22}$ Cf. L. Landgrebe, «Die Zeitanalyse in der Phänomenologie und in der klassischen Tradition ", in Phänomenologie - lebendig oder tot? Zum 30. Todesjahr Edmund Husserls, E. Fink et al. (éd.), Karlsruhe, Badenia Verlag, 1969.

${ }^{23} \mathrm{~K}$. Held, Lebendige Gegenwart. Die Frage nach der Seinsweise des transzendentalen Ich bei Edmund Husserl, entwickelt am Leitfaden der Zeitproblematik, Phaenomenologica, ${ }^{\circ} 23$, La Haye, Martinus Nijhoff, 1966, p. 149. Voir A. Schütz, Collected Papers I, M. Natanson (éd.), Phaenomenologica, $\mathrm{n}^{\circ}$ 41, La Haye, Martinus Nijhoff, 1962, p. XXXI.
} 
profonde de la possibilité de toutes les fonctions et de leurs effectuations.

Les fonctions constitutives sont toujours des «pouvoirs de...». Cependant, des pouvoirs ne sont «là » pour nous que pour autant qu'ils sont exercés. Lorsque ce fonctionnement devient lui-même objet de réflexion - réflexion qui n'est jamais qu'une prise de conscience après coup -, il n'est plus saisi comme ce qu'il est dans son accomplissement vivant. C'est uniquement dans un tel accomplissement qu'il est "lui-même là ». Sans doute cette manière apodictiquement certaine d'être "soi-même là » ne répond-elle pas aux exigences formulées par Husserl en matière de retour à la certitude apodictique ultime. "Être soi-même là » signifie pour Husserl être intuitionné dans la réflexion phénoménologique. Mais une fonction entendue comme un pouvoir ne peut être intuitionnée. Son "être soimême là » ne peut être appréhendé que dans son exercice. On touche là assurément à une limite de la réflexion en tant que prise de conscience après coup et thématisation de ce qui est déjà aperçu, limite en deçà de laquelle se trouve néanmoins quelque chose d'apodictiquement et immédiatement certain. Ce quelque chose est à coup sûr pré-langagier. Aussitôt qu'il est verbalement formulé, il se voit soumis à la contrainte de l'ontification qui est le corollaire inévitable du discours, puisque la signification des mots dans lesquels le discours s'articule se réfère toujours déjà à des éléments préalablement constitués et non à des fonctions ultimement constituantes. Cependant, ce Là absolu n'est pas simplement déduit ; il est au contraire, dans son fonctionnement même, familier et certain : [38] certain comme le Là inéluctable par lequel nous sommes par avance toujours posés, et à l'égard duquel nous sommes contraints de prendre position. En ce sens, il est un factum absolu qu'on ne peut qu'admettre et qui ne saurait être davantage interrogé par la réflexion. «Le Je ultimement fonctionnant, en tant que Je stable, ne peut se rattraper lui-même. Le Je ultimement fonctionnant précède constamment sa propre présentation réflexive $»^{24}$.

Telle est, très schématiquement exposée, la voie génétique de la réflexion de la monade égoïque méditant transcendantalement. Cette voie conduit à son Là imprescriptible comme fondement ultime de la certitude apodictique de soi-même en tant que présent fonctionnel vivant. Ce fondement lui-même ne peut plus être thématisé réflexivement. Il est bien plutôt éprouvé comme limite sur laquelle vient buter la réflexion transcendantale et contre laquelle elle se brise. Mais si la réflexion a bien en premier lieu le sens d'une réflexion sur un pou-

${ }^{24}$ K. Held, Lebendige Gegenwart, op. cit., p. 131. 
voir, cela signifie qu'elle bute ici sur la limite de son pouvoir, face à laquelle tout pouvoir est impuissant et qu'il doit tout simplement admettre. Ainsi, dans l'accomplissement de ses fonctions constitutives, chaque sujet égoïque se trouve immédiatement posé en son Là - ou, pour le dire dans la langue du Sein und Zeit de Heidegger, « jeté » dans son Là. Dans cet accomplissement, il devient immédiatement familier avec lui-même comme centre de fonctions ainsi qu'avec leur pouvoir. Il s'éprouve lui-même à travers ce dont il est capable, et fait d'entrée de jeu l'expérience de son pouvoir comme quelque chose qui a dû être éduqué et exercé. Or, cette expérience implique déjà la référence à l'histoire de sa vie : le pouvoir, en lequel le sujet s'éprouve lui-même, est lui-même éprouvé comme étant devenu un tel pouvoir, et cela grâce à l'histoire des expériences subjectives à travers lesquelles ce pouvoir s'est formé. De façon élémentaire, le sujet égoïque peut se familiariser réflexivement avec cette histoire au titre de l'histoire de sa vie ; [39] elle a néanmoins toujours déjà eu lieu et produit ses effets " anonymement », avant toute réflexion sur elle. Une telle réflexion peut être exprimée verbalement, en demandant par exemple : "Pourquoi est-ce arrivé ainsi, pourquoi ai-je fait cela ? » Aussi Husserl peut-il dire : " La question du pourquoi est originellement question de $1^{\prime \prime h}$ histoire" $»^{25}$. En d'autres termes, elle est la question de $l^{\prime}$ « historicité intrinsèque », historicité qui précède toute remémoration explicite et toute autobiographie, et qui est le fondement transcendantal de leur possibilité.

Cette " historicité intrinsèque » peut être trouvée sur la voie d'une réflexion "égologique » et d'une question en retour d'ordre génétique. Elle est alors découverte comme l'historicité de la vie chaque fois propre, distincte de la vie d'autrui par son Là imprescriptible et inéchangeable. Or, selon Husserl, cette historicité intrinsèque doit être $l^{\prime}$ « a priori $»$ de $l$ 'histoire, que celle-ci soit entendue comme histoire de sa propre famille, de son propre peuple, de sa propre époque, ou encore, de manière tout à fait générale, comme histoire de $l^{\prime}$ « humanité »-donc toujours de $l$ 'histoire que chacun a en commun avec les autres. Comment dès lors les autres peuvent-ils pénétrer dans mon «historicité intrinsèque» propre, ou bien, s'ils y sont toujours déjà, comment mettre en évidence ce fait? Cette question n'est autre que celle de la "fenêtre » monadique. C'est seulement si l'existence d'une telle fenêtre peut être attestée - non pas comme quelque chose que l'on se contenterait de déduire ou de postuler, mais comme quelque chose dont on fait d'emblée l'expérience - que

${ }_{25}$ Hua XV, Nr. 24, p. 420 [Sur l'intersubjectivité II, trad. N. Depraz, Paris, PUF, 2001, p. 341]. 
l'historicité intrinsèque chaque fois propre peut en effet être revendiquée comme a priori de l'histoire, et qu'il devient possible de comprendre l'entre-appartenance des deux thèses de l'absoluité de la monade et de l'absoluité de l'histoire.

[40] Frayer la voie vers une réponse à ces questions: telle est la tâche de la théorie transcendantale génétique de l'intersubjectivité. Comme nous l'avons déjà noté, le premier exposé détaillé de la théorie husserlienne de l'intersubjectivité, dans la cinquième Méditation cartésienne, s'était soldé par un échec du fait que Husserl cherchait à y développer cette théorie sur un mode statique. Nous revenons sur ce point, en nous bornant à donner quelques indications (une enquête détaillée déborderait largement le cadre du présent propos). Les trois volumes désormais disponibles contenant les analyses husserliennes de l'intersubjectivité (Husserliana XIII-XIV-XV) ${ }^{26}$ attestent que Husserl a dépassé dès 1927 le cadre de l'analyse purement statique, en cherchant à reconduire la constitution de l'intersubjectivité par aperception apprésentante et analogisante à sa pré-constitution passive dans une aperception instituée associativement. Mais cette tentative elle-même laisse ouverte la question de savoir si une telle analogisation associative ne présuppose pas déjà l'auto-différenciation de la monade visà-vis du Tu, du Nous et, de manière générale vis-à-vis de l'autre, de sorte que son propre Là puisse correspondre avec le Là de l'autre ; donc la question de savoir si la temporalité et l'historicité intrinsèques qui lui sont chaque fois propres ne seraient pas a priori en relation avec la temporalité et l'historicité de l'autre, de sorte que devienne possible un temps commun et que nous soyons autorisés à parler d'une « intersubjectivité originaire» qui serait co-originaire du «Je originaire» chaque fois singulier, si bien que l'un ne puisse aller sans l'autre. Ainsi, à la question: "Comment les autres pénètrent-ils dans ma propre temporalisation originaire? $\gg^{27}$, il conviendrait de répondre: d'une manière telle que chaque "âme" " est certes en soi et pour soi, mais n'a pourtant de sens que [41] dans une pluralité enracinée en ellemême et devant se déployer à partir d'elle-même $»^{28}$.

Husserl n'a plus eu l'occasion de développer systématiquement ces questions et de leur apporter une réponse. Certains de ses manuscrits tardifs indiquent néanmoins dans quelle direction une telle réponse peut être cherchée. Husserl y reprend sa caractérisation

\footnotetext{
${ }^{26}$ Voir l'analyse détaillée que propose S. Strasser de l'ontologie sociale de Husserl («Grundgedanken der Sozialontologie Edmund Husserls ", Zeitschrift für philosophische Forschung, n 29, 1975, p. 3-33), qui n'était pas encore publiée lors de la rédaction de cette contribution.

${ }^{27}$ Hua XV, Beilage XX, p. 353. [La phrase exacte n'a pu être retrouvée. [N. $d$. T.]]

${ }^{28}$ Hua XV, Nr. 20, p. 341 [Sur l'intersubjectivité II, op. cit., p. 142.]
} 
antérieure de l'intentionnalité comme «aspiration originaire de la monade», en lui donnant une forme plus concrète : "Ne pouvonsnous pas ou ne nous faut-il pas supposer une intentionnalité pulsionnelle universelle, qui constitue unitairement tout présent originaire comme temporalité stable, et qui concrètement pousse de présent en présent, de sorte que tout contenu soit contenu de remplissement pulsionnel et soit visé avant le but ? »29

L'analyse structurelle du présent originaire (c'est-à-dire du fluer vivant stable) nous conduit à la structure égoïque, et à la couche sous-jacente fondatrice de cette structure, à savoir la couche du fluer non-égoïque; avec ce fluer, nous sommes reconduits, à la faveur d'un questionnement en retour sur ce qui constitue et ce que présuppose toute activité sédimentée, à une dimension radicalement pré-égoïque ${ }^{30}$.

C'est en ce sens que Husserl comprend la pulsion sexuelle comme « intentionnalité originaire instinctive de communautisation $»^{31}$. En tant que pulsion originaire, elle se rapporte d'emblée à l'autre et trouve en celui-ci sa réplique : «Dans la pulsion même gît le fait de se rapporter à l'autre et à sa pulsion corrélative ». Dans son « remplissement sur son mode originaire, nous n'avons pas deux remplissements s'accomplissant séparément, à savoir dans une primordialité et dans l'autre, mais une unité des deux primordialités se produisant par $l^{\prime}$ entrelacs des remplissements $»^{32}$. [42]

Essayons de compléter ces idées en les prolongeant quelque peu.

Le remplissement d'une «pulsion originaire » peut avoir pour conséquence la naissance d'un nouvel être humain, avec une histoire qui lui est alors propre, mais qui est déjà largement déterminée quant à ses possibilités par l'héritage de ses ascendants ${ }^{33}$. Ainsi, la procréation et la naissance ne sont pas seulement des thèmes de la biologie, mais possèdent également une signification philosophico-transcendantale, en tant que conditions de possibilité de l'histoire. En elles se fonde la différence des générations, à travers laquelle ce qu'il y a de commun à ces générations apparaît selon des perspectives différentes

\footnotetext{
${ }^{29}$ Hua XV, Nr. 34, p. 595 [« Téléologie universelle », trad. J. Benoist, Philosophie, n 21, 1989, p. 4].

${ }^{30}$ Hua XV, Beilage XLIII, p. 598].

${ }^{31}$ Ms. E III 10, p. 10. [Voir à présent E. Husserl, Grenzprobleme der Phänomenologie. Analysen des Unbewusstseins und der Instinkte. Metaphysik. Späte Ethik. Texte aus dem Nachlass (1908-1937), Hua XLII, R. Sowa et T. Vongehr (éd.), Dordrecht, Springer, 2014, Nr. 6, p. 97].

32 Hua XV, Nr. 34, p. 593-594 [« Téléologie universelle», op. cit., p. 3].

${ }^{33}$ Sur le problème de $l^{\prime}$ « héritage » comme horizon vide, voir Hua XV, Beilage XLV, p. 604 [«L'enfant - La première Einfühlung», trad. N. Depraz, Alter. Revue de phénoménologie, n 1 , 1993, p. 265].
} 
du fait de leurs temps de vie distincts. C'est ainsi que se constitue une histoire commune, celle d'une famille, d'un peuple, d'une époque, etc. Chaque maillon de cette histoire, $s^{\prime} y$ insérant par sa naissance et sa croissance, possède son temps qui lui est alloué. Seul un tel retour aux structures génétiques les plus profondes (qui n'ont pu ici être abordées qu'allusivement) permet de donner à la proposition suivante de Husserl un contenu concret: «L'histoire n'est d'entrée de jeu rien d'autre que le mouvement vivant d'interaction et d'implication réciproques entre la formation de sens et la sédimentation de sens originaires $»^{34}$.

Les «fenêtres » de la monade égoïque ne doivent donc pas seulement être cherchées en haut, dans l'aperception ou, plus profondément, dans l'analogisation associative, mais dans la "pulsion originaire » qui, en tant que telle, est toujours déjà rapportée à l'autre et trouve en lui sa réplique et sa satisfaction. C'est sur cette union originaire dans la duplicité que se fonde la possibilité de l'analogie entre une co-présence et une présence en personne, telle qu'elle a été thématisée par Klaus Held dans sa confrontation déjà citée avec la théorie husserlienne de l'intersubjectivité. La certitude à propos du Là de [43] l'autre précède donc toute aperception et toute association. Et elle n'a pas non plus son fondement ultime dans la relation Je-Tu telle qu'elle a été étudiée par Bernhard Waldenfels ; celui-ci en avait $\mathrm{du}$ reste lui-même parfaitement conscience, puisqu'il en est finalement venu à poser la question $\mathrm{du}$ "soubassement» du dialogue ${ }^{35}$. Cette unité préalable dans la séparation absolue est éprouvée de manière originairement passive dans l'accomplissement de l'aspiration dirigée sur l'autre, qui trouve sa réplique en celui-ci. Cette expérience est une "expérience absolue ${ }^{36}$, et c'est sur le fondement de celle-ci que l'histoire est absolue. Mais cette histoire est alors davantage que la res gestae, en ce sens qu'elle porte en elle ce qui précède toute res gestae, à savoir la structure transcendantale de l'être-un dans la séparation, laquelle conduit ensuite à un nouvel être-un, à une nouvelle monade absolue portant en elle les caractères génératifs communs. Ces caractères sont communs, dans la mesure où le remplissement de l'intentionnalité originaire pulsionnelle a produit une «unité des deux primordialités », à savoir celle de l'un et celle de l'autre. Chacun possède dans son accomplissement fluant son propre Là et, dans le remplissement,

\footnotetext{
34 Hua VI, p. 380 [La crise des sciences européennes et la phénoménologie transcendantale, op. cit., p. 420].

${ }^{35}$ Cf. B. Waldenfels, Das Zwischenreich des Dialogs. Sozialphilosophische Untersuchungen in Anschluss an Edmund Husserl, Phaenomenologica, ${ }^{\circ}$ 41, La Haye, Martinus Nijhoff, 1971.

36 Cf. L. Landgrebe, "The phenomenological Concept of Experience», Philosophy and Phenomenological Research, vol. 34, 1973, p. 1-13.
} 
celui-ci devient un Là commun. Ce Là est le Là des sujets qui se meuvent corporellement. C'est dans leur se-mouvoir corporel que s'ouvre d'emblée à eux une "nature », dans la mesure où, en tant que sujets corporels, ils « appartiennent » eux-mêmes à la nature. Un Là a sa « place » dans la nature, et il est ainsi en relation avec la «Terre ». Celle-ci «s'inscrit dans une "histoire naturelle" universelle, au sein de laquelle l'homme se développe comme être vivant $»^{37}$.

Mais, pour autant, l'histoire ne se réduit aucunement à l'histoire naturelle. Elle a assurément pour sol le "processus naturel», mais non pas au sens d'une "matière » impropre à recevoir d'autres déterminations, mais au sens de ce qui nous est familier en tant que "Terre », c'est-à-dire comme l'ensemble des conditions assignant à l'activité de l'homme, et ainsi à son histoire au [44] sens de la res gestae, leurs limites. Telle est précisément la situation en laquelle nous faisons aujourd'hui l'expérience de l'histoire : cette limitation de toutes les possibilités humaines est devenue d'autant plus expressément consciente que le processus de domination de la Terre est avancé. À l'absoluité de notre Là ressortit l'absoluité de la Terre comme limite de tout Là possible. Nous n'aborderons pas ici la question du voyage dans l'espace. Un tel voyage pourra difficilement avoir lieu autrement que "référé à la Terre ». En ce sens, la "Terre » est donc un concept transcendantal limite. Elle est une structure fondamentale du monde-de-lavie humain et désigne en même temps la limite par rapport à laquelle seulement tout discours sur la «nature » peut recevoir un sens remplissable intuitivement. Et ce concept limite coupe court à toute spéculation sur un règne de la nature en soi. Il n'y a d'histoire de la Terre et d'histoire de la nature que parce que, en vertu de notre individuation, nous sommes des êtres historiques, et que toute détermination temporelle $\mathrm{du}$ processus naturel renvoie ultimement au Là particulier de tels êtres historiques.

$C^{\prime}$ est en ce sens universel, incluant également le processus de la nature, que doit être compris le mot de Husserl sur l'histoire comme fait majeur de l'être absolu. Il s'agit du processus fluant stable, de la "vie transcendantale", laquelle ne s'éprouve comme processus que parce qu'elle s'accomplit toujours déjà comme séparation et en même temps unification des centres de mouvements (des «monades ») qui se meuvent d'abord anonymement ${ }^{38}$. C'est en ce sens qu'il faut com-

\footnotetext{
${ }^{37}$ Ms. B III 1, p. 34-35, cité d'après B. Waldenfels, op. cit., p. 335, note 47. Voir aussi Hua XV, Nr. 38 ["Temporalisation - monade", trad. S. Margel, Études phénoménologiques, n 19, 1994, p. 3-10] et Hua VI, p. 304 [La crise des sciences européennes et la phénoménologie transcendantale, op. cit., p. 336].

${ }^{38}$ Hua XV, Beilage XXII, p. 373 sq. [Sur l'intersubjectivité II, op. cit., p. 553 sq.].
} 
prendre que «le Je est ce qui précède tout le reste, mais dans la précédence du fluer lui-même $»^{39}$. Par là, on peut également comprendre le sens de la proposition selon laquelle la «nature » ellemême est une «formation de [45] $1^{\prime}$ histoire transcendantale $»^{40}: c^{\prime}$ est seulement parce que les sujets transcendantaux, les monades, sont déjà " historiques » qu' « il y a » pour nous quelque chose comme une histoire de la nature et, par suite, une science de la nature (laquelle n'a pu se constituer en son sens moderne qu'en faisant méthodiquement abstraction de cette référence transcendantale). C'est par conséquent en ce sens qu'il faut comprendre l'entre-appartenance de l'absoluité de l'ego et de l'absoluité de l'histoire. Dans quelle mesure la détermination de $\mathrm{l}^{\prime}$ " absolu » comme «Là » est-elle alors une détermination transcendantale et apriorique, et par suite la phénoménologie, en tant qu'elle reconduit à ce point suprême, une philosophie transcendantale? Pour autant que cette détermination conduit à ce qui précède toujours déjà tout ce dont nous pouvons dire avec quelque sens qu' «il est». La facticité de ce Là est un principe structurel ${ }^{41}$. Le précéder est, par son antériorité même, "anonyme ». Si bien que " anonyme » et "Là » sont des déterminations transcendantales avec lesquelles nous atteignons le point ultime de la réflexion transcendantale. C'est le point où cette réflexion trouve la limite qu'elle se fixe elle-même et qu'elle ne peut dépasser. Elle ne peut remonter en deçà de cette précédence. Cette découverte met donc au jour le foyer à partir duquel la réflexion transcendantale et la philosophie transcendantale revêtent le sens d'une critique de tout dogmatisme. Une telle critique n'est pas seulement critique de la métaphysique ancienne, pré-kantienne, mais aussi critique des implications métaphysiques cachées dans les positions modernes se croyant antimétaphysiques. Elle interdit également de transgresser ces limites, qu'elle a elle-même trouvées, en recourant à une «doctrine des postulats » au sens de Kant. Mais, pour autant, elle ne conduit pas à un scepticisme absolu. Car, en considérant justement les conditions de possibilité pour $\mathrm{qu}^{\prime}$ « il y ait» pour nous quelque chose comme $l^{\prime}$ « histoire», elle conduit à la vision évidente de ce qui est exigé de nous, d'une manière inconditionnellement universelle et contraignante, comme principe suprême de la conduite de la vie, étant donnée cette condition humaine ${ }^{42}$ et ses limites caractéristiques. Nous avons essayé de montrer que la facticité de l'histoire

\footnotetext{
${ }^{39}$ Ms. C 7 I, 18.

${ }^{40}$ Hua XV, Nr. 23, p. 391 [Sur l'intersubjectivité I, op. cit., p. 324].

${ }^{41}$ Cf. A. Aguirre, Genetische Phänomenologie und Reduktion. Zur Letztbegründung der Wissenschaft aus der radikalen Skepsis im Denken E. Husserls, op. cit.

42 En français dans le texte. [N. $d . T$.]
} 
dépend de la facticité du Là de l'existence individuelle chaque fois singulière ${ }^{43}$. À supposer que cette humanité, rivée à la «Terre » dans son Là, ait encore un avenir, et que l'histoire ne soit pas parvenue à son terme, la condition pour qu'il y ait encore quelque chose comme l'histoire ne serait pas seulement la reconnaissance de nos "semblables » comme nous étant justement semblables, mais aussi la reconnaissance de ceux-ci comme étant individuellement absolument différents. Ce qui est exigé de manière inconditionnellement universelle et contraignante, c'est donc la reconnaissance et le respect de l'existence individuelle chaque fois propre de chacun de nos "semblables ", ainsi que des groupes historiquement advenus tels que peuples, ethnies, nations, etc., dans leur individualité « collective». Ce n'est qu'avec cette reconnaissance qu'assumer sa propre facticité peut légitimement signifier non pas la soumission à un destin aveugle, mais bien au contraire la possibilité d'un monde humain.

Traduit de l'allemand par Guillaume Fagniez et John Tryssesoone

${ }^{43}$ Cf. L. Landgrebe, «Faktizität und Individuation », in Sein und Geschichtlichkeit. Festschrift für K. H. Volkmann-Schluck, W. Janke (éd.), Francfort-sur-le-Main, Klostermann, 1974, p. 275 sq. 